\title{
La révolution des Linked Open Data en numismatique : Les exemples de nomisma.org et Online Greek Coinage
}

\author{
The Linked Open Data Revolution in Numismatics: The examples of \\ nomisma.org and Online Greek Coinage
}

\author{
David Wigg-Wolf ${ }^{1}$, Frédérique Duyrat ${ }^{2}$
}

${ }^{1}$ Römisch-Germansiche Kommission des Deutschen Archäologischen Institut Palmengartenstrasse, Frankfurt, Allemagne, david.wigg-wolf@dainst.de

${ }^{2}$ Directrice du département des Monnaies, médailles et antiques Orient et Méditerranée - Mondes sémitiques (UMR 8162), Bibliothèque nationale de France, frederique.duyrat@bnf.fr

RÉSUMÉ. Le projet nomisma.org a été lancé par l'American Numismatic Society en 2010. A l'origine, l'objectif était de faciliter la présentation en ligne de concepts numismatiques en employant les méthodes du Web sémantique ou Linked open data. Ce faisant, nomisma.org a construit les bases de l'échange de données entre machines.

Les projets qui emploient les concepts de nomisma.org, tels que OCRE et CRRO, peuvent à présent être utilisés aussi bien par des cabinets des médailles et des inventaires de trouvailles archéologiques dans le bus d'échanger des données que pour publier ces données en ligne. Cet article se présente en deux parties. La première est consacrée à une illustration pratique de la manière dont les données sont liées par nomisma.org afin de montrer ce qui est possible aujourd'hui. Elle présente aussi comment ce but est atteint et l'ontologie qui sous-tend le projet. L'expérience nomisma.org fournit des modèles qui peuvent être utilement employés pour d'autres matériels archéologiques. La seconde partie est consacrée aux développements récents en numismatique grecque et à la structuration des données qui les sous-tend.

ABSTRACT. The project nomisma.org was initiated by the American Numismatic Society in 2010. The original aim was to facilitate the online presentation of numismatic concepts employing the methods of the Semantic Web and Linked Open Data. In this way nomisma.org laid the foundation for the machine-based exchange of data between systems.

Projects that employ the concepts of nomisma.org, such as OCRE and CRRO, can now be used by both coin cabinets and inventories of archaeological coin finds in order to exchange data, as well as to publish them online. This paper consists of two parts; in the first part the emphasis is placed on the one hand on a practical illustration of how the data are linked by nomisma.org in order to show just what is possible at present. But it will also explain how this is achieved and present the underlying ontology. The experience of nomisma.org provides useful lessons that can be applied productively to other archaeological material. The second part is devoted to recent developments in Greek numismatics and the underlying data structure.

MOTS-CLÉS. Numismatique, Linked open data, web sémantique, RDF.

KEYWORDS. Numismatics, Linked open data, Semantic web, RDF.

\section{Introduction}

Cette contribution présente des exemples des progrès significatifs accomplis ces dernières années dans l'application des Linked open data à la numismatique. Les monnaies sont particulièrement adaptables aux Linked open data car ce sont des objets sériels produits en masse et leurs données descriptives de base sont relativement simples.

L'article est formé de deux parties. Le projet nomisma.org, base de l'application des Linked open data à la numismatique, est présenté dans une première partie ainsi que plusieurs projets de numismatique romaine qui emploient les concepts de nomisma.org pour exploiter, présenter et analyser les données. La seconde partie propose un certain nombre d'exemples pratiques tirés des récents développements concernant la monnaie grecque. En effet, les projets romains sont fondés sur l'existence de catalogues de référence qui peuvent servir de base à la création d'ontologies, de (C) 2017 ISTE OpenScience - Published by ISTE Ltd. London, UK - openscience.fr 
vocabulaires standardisés et d'outils de recherche adaptés. Au contraire, les monnaies grecques, frappées pendant près de mille ans par près de deux mille ateliers offrent un paysage trop complexe pour qu'il ait jamais existé un unique catalogue de référence. Les Linked open data sont sans doute la solution pour créer un outil de référence.

\section{1. nomisma.org et Linked open data}

Pour être insérées dans de Linked open data, les données doivent répondre aux exigences des cinq étoiles du LOD évoquées par Tim Berners-Lee, le créateur du World Wide Web.

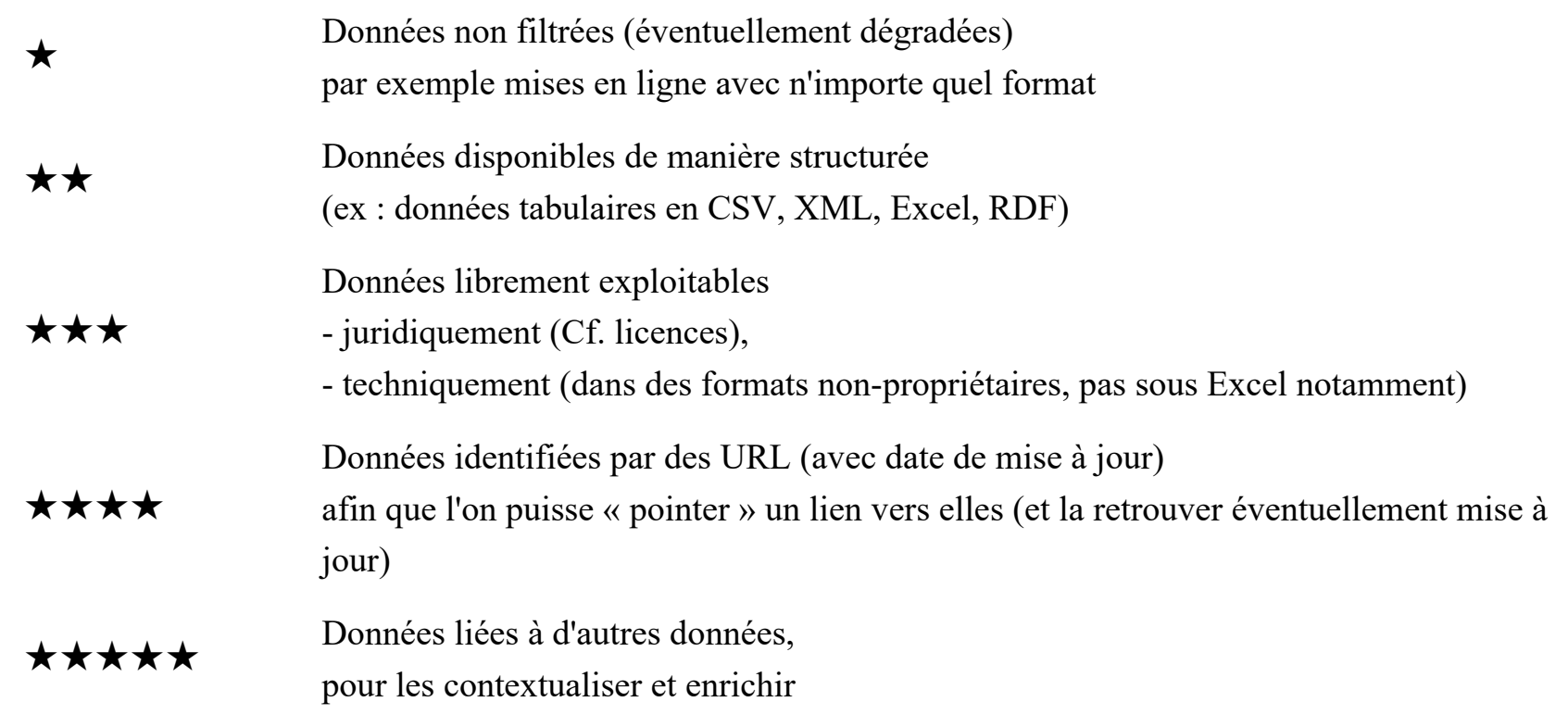

Le but du projet nomisma.org est «de fournir les représentations digitales stables de concepts numismatiques selon les principes des Linked open data » et de fournir les outils nécessaires pour incorporer les données numismatiques au Web sémantique. Le projet a été initié par l'American Numismatic Society et l'Institute for Advanced Studies of the Ancient World de New York. C'est maintenant l'œuvre d'une coopération internationale impliquant des partenaires de partout dans le monde, incluant l'Université Paris-Sorbonne, le British Museum, le Deutsches Archäologisches Institut, le Cabinet des médailles des Staatliche Museen zu Berlin, l'université Goethe de Frankfurt am Main et la Bibliothèque nationale de France.

Deux éléments fondamentaux forment la base du projet nomisma. Le premier est constitué par les URIs, Uniform Resource Identifiers, en d'autres termes, une adresse Web unique et permanente attribuée à chaque définition de concept numismatique disponible en ligne. Par exemple http://nomisma.org/id/denarius est l'URI nomisma pour le denier, la monnaie romaine d'argent de la République romaine et du Haut Empire. Un autre exemple dans nomisma pourrait être http://nomisma.org/id/tiberius, l'URI pour l'empereur romain Tibère. Elle fournit une définition d'Auguste ainsi que des liens avec des références dans d'autres ressources, comme par exemple l'URI de la BnF pour Tibère. 


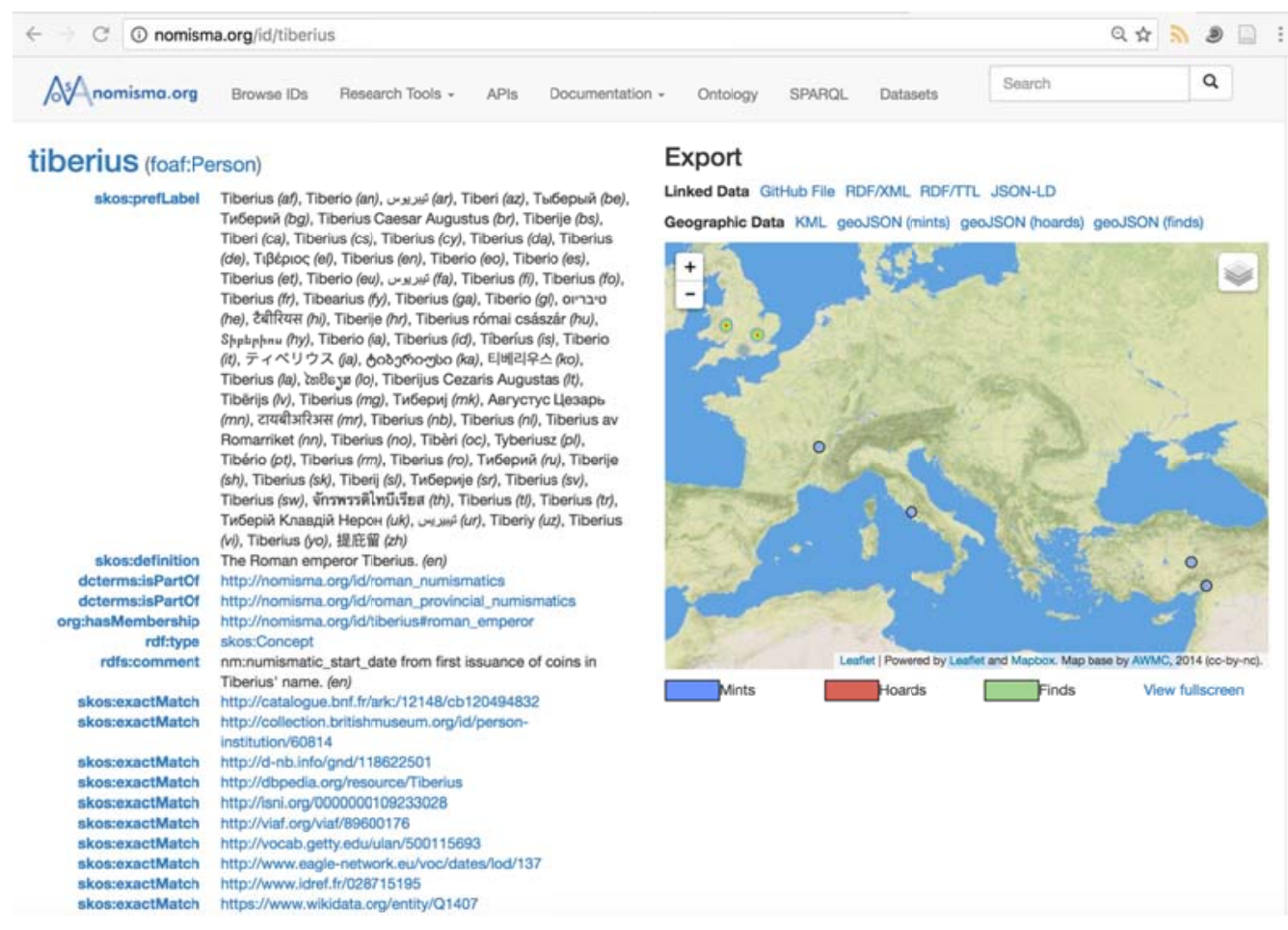

Figure 1. Page nomisma.org pour l'empereur romain Tibère : le lien pour l'entrée de la BnF pour Tibère est signalé dans la liste de liens (http://nomisma.org/id/tiberius)

Des objets numismatiques peuvent aussi avoir des URIs sans être dans nomisma. Par exemple http://numismatics.org/collection/1985.140.20 est l'URI de la monnaie portant le numéro d'inventaire 1985140.20 dans les collections de l'American Numismatic Society. C'est à travers de tels liens avec d'autres ressources que nomisma s'intègre au monde des Linked open data et du Web sémantique. Ainsi, nomisma.org, comme d'autres ressources telles que AFE, OCRE et CRRO, est lié à divers thesauri, référentiels et systèmes comme le Getty Art and Architecture Thesaurus (http://www.getty.edu/research/tools/vocabularies/aat/), Pleiades (https://pleiades.stoa.org/), Geonames (http://www.geonames.org/), Zenon (http://zenon.dainst.org/), et le iDAI.gazetteer (https://gazetteer.dainst.org/app/\#!/home), ainsi que d'autres ressources similaires largement utilisées qui sont incorporées dans le but de lier les données numismatiques avec d'autres informations archéologiques. 


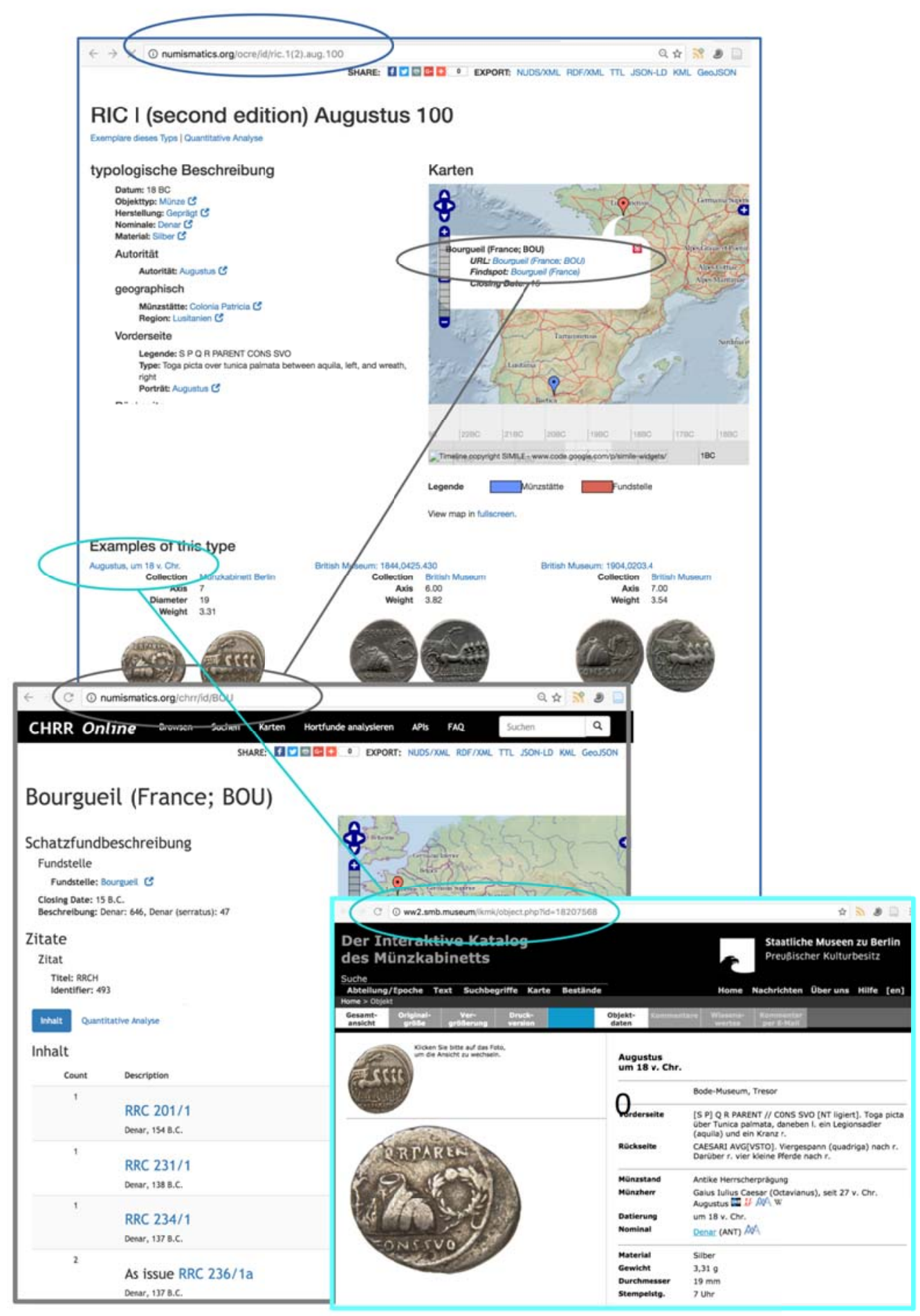

Figure 2. Comment OCRE, CHRR et une monnaie de Berlin sont liés en utilisant les Linked open data (http://numismatics.org/ocre/id/ric.1(2).aug.100; http://ww2.smb. museum/ikmk/object.php?id=18207568; http://numismatics.org/chrr/id/BOU).

La figure 2 illustre la manière dont les ressources peuvent être liées. Le site web Online Coins of the Roman Empire (OCRE) fournit des représentations numériques des types monétaires dans le corpus de référence sur les monnaies frappées par les empereurs romains (http://numismatics.org/ocre/). L'image du haut montre l'entrée pour le type RIC 100 frappé par l'empereur Auguste. Dans OCRE, cette entrée porte l'URI http://numsimatics.org/ocre/id/ric.1(2).aug.100 visible en haut de la figure. Cette adresse n'est pas seulement lisible par les machines mais est aussi compréhensible pour les humains. "ric.1(2)" indique que la référence est la seconde édition du volume 1 du Roman Imperial Coinage (RIC), "aug" 
représente Auguste, "100" est le numéro de l'entrée dans le corpus. En plus de la description du type visible sur la gauche, la page Web montre une sélection de monnaies du même type provenant de différentes collections et ressources, dans le cas présent trois monnaies. Celle représentée à gauche est de la collection du Staatliche Museen zu Berlin. Dans OCRE, la notice de cette monnaie comprend un lien hypertexte dirigeant l'utilisateur sur le site de la collection de Berlin par le biais de l'URI de la monnaie sur le site internet de la collection d'origine. L'entrée de cette monnaie sur le site internet de Berlin est visible dans la barre d'adresse de l'image en bas à droite.

L'entrée OCRE inclut aussi une carte montrant les trouvailles de monnaies de ce type, dans le cas illustré, le trésor monétaire de Bourgueil. L'étiquette du lieu de trouvaille sur la carte est liée à une autre ressource enrichie par nomisma.org: le site du projet Coin Hoards of the Roman Republic (CHRR) (http://numismatics.org/chrr/), dans l'image en bas à gauche. Le lien depuis OCRE est fourni par l'URI du trésor de Bourgueil dans CHRR, http://numismatics.org/chrr/id/BOU. La page internet dans CHRR fournit des informations détaillées sur les monnaies appartenant à ce trésor. Leurs types sont en retour liés à un autre site internet, non représenté dans la figure 2, Coins of the Roman Republic Online (CRRO) (http://numismatics.org/crro/), un corpus des types frappés durant la République romaine analogue à OCRE.

Mais nomisma nous permet de faire mieux que seulement fournir des URIs pour relier des sites web entre eux. Nomisma a aussi produit une ontologie numismatique. Qu'est-ce exactement qu'une ontologie ? À quoi sert-elle ? Un parallèle avec le langage rend ce concept facilement compréhensible : d'une part, nous avons un vocabulaire avec des mots tels que Tibère ou denier présentés sous la forme d'URIs nomisma. L'ontologie, d'autre part, fournit les éléments et la grammaire qui nous permet d'assembler les mots pour former des phrases qui aient du sens. Parmi les éléments de l'ontologie, « hasDenomination »permet d'associer la valeur à la monnaie, ou « hasAuthority », relie la monnaie à la personne qui était responsable de sa production.

Les URIs nomisma et l'ontologie peuvent alors être utilisées pour construire des phrases sur le denier de Berlin qui apparaît dans la figure 2 .

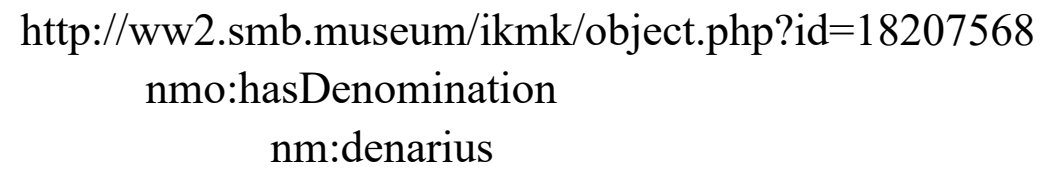

http://ww2.smb.museum/ikmk/object.php?id=18207568

nmo:hasDenomination

nm:denarius

http://ww2.smb.museum/ikmk/object.php?id=18207568

nmo:hasAuthoriy

nm:augustus

Constituées de trois parties, de telles phrases sont appelées des triplets dans ce langage informatique appelé RDF, Resource Description Framework. Si nous, humains, pouvons comprendre ces triplets - la dénomination de la monnaie est le denier, l'autorité qui a frappé cette monnaie est Auguste - de telles phrases peuvent aussi être comprises par les ordinateurs. Le RDF nous permet de lier les ressources et d'échanger des informations entre ordinateurs et sites web de façon très complexe.

Généralement, les données sont conservées dans des bases de données relationnelles et les données de la monnaie de Berlin devraient être conservées comme le montre la figure 3. L'entrée principale de la monnaie doit être liée aux entrées du denier et d'Auguste dans les tableaux donnant les listes de dénominations et d'autorités dans la base de données. 


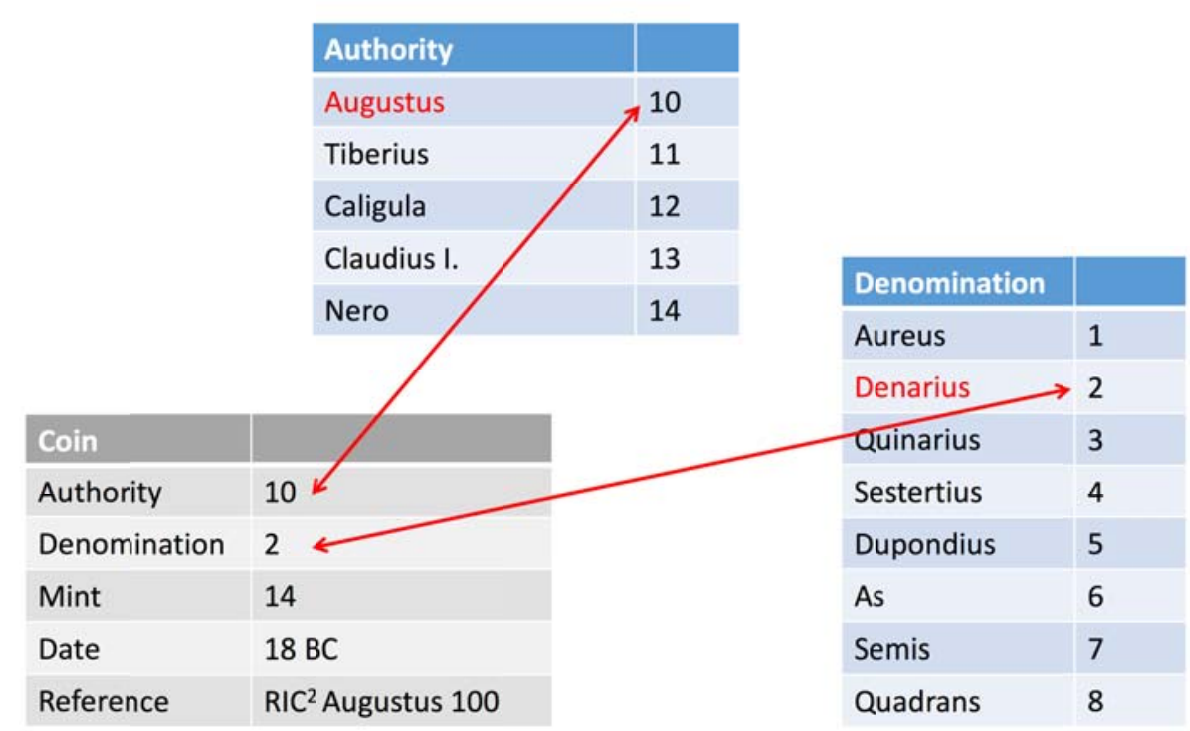

Figure 3. Structure d'une base de données relationnelles classique pour les champs autorités et dénominations (K. Tolle).

L'échange de données et la communication entre des bases de données relationnelles pourraient être très complexes et difficiles si ces bases n'ont pas des structures similaires. Mais l'utilisation du RDF rend cette communication bien plus facile. Si des bases de données, y compris des bases avec des structures très différentes, peuvent être mappées en RDF, alors elles s'expriment dans un format RDF commun, et selon l'ontologie nomisma pour les monnaies. Il est dès lors possible de fournir un accès commun à des données provenant de ressources très disparates. L'idée derrière ce processus est représentée dans la figure 4. Les données des différentes bases sont toutes converties en RDF et sont donc de ce fait requêtables par un point d'accès commun ("generic portal").

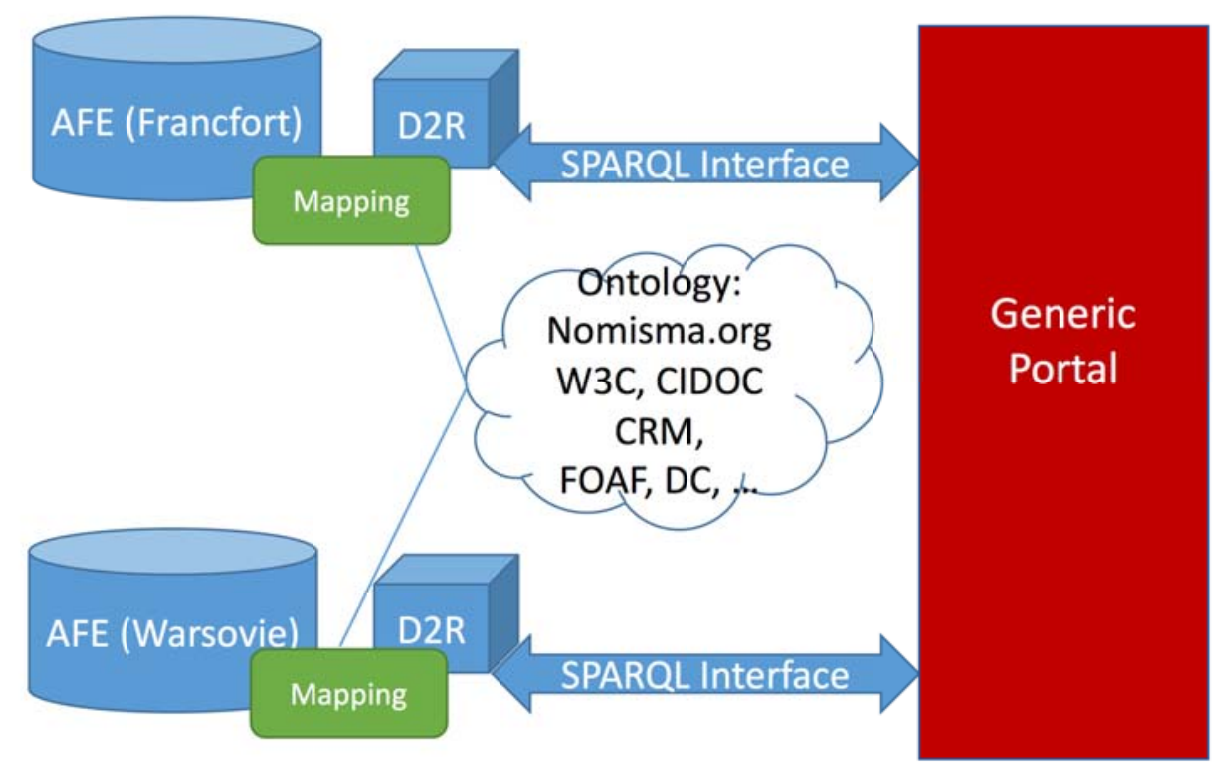

Figure 4. Représentation schématique de la conversion des données issues des bases relationnelles de Francfort et Varsovie vers un format RDF commun (K. Tolle).

L'accès au RDF est possible en utilisant le langage de requête SPARQL, comme le montre la figure 5. Dans ce cas, un SPARQL-Endpoint (point d'accès commun), développé pour le projet Antike Fundmünzen in Europa (AFE) (http://afe.fundmuenzen.eu/) par la Römisch-Germanische Kommission et l'université Goethe de Francfort, requête deux bases de données à Francfort et Varsovie avec des 
informations sur les trouvailles monétaires en Allemagne et en Pologne. Les bases de données ont été mappées en RDF en utilisant l'ontologie et le vocabulaire nomisma.

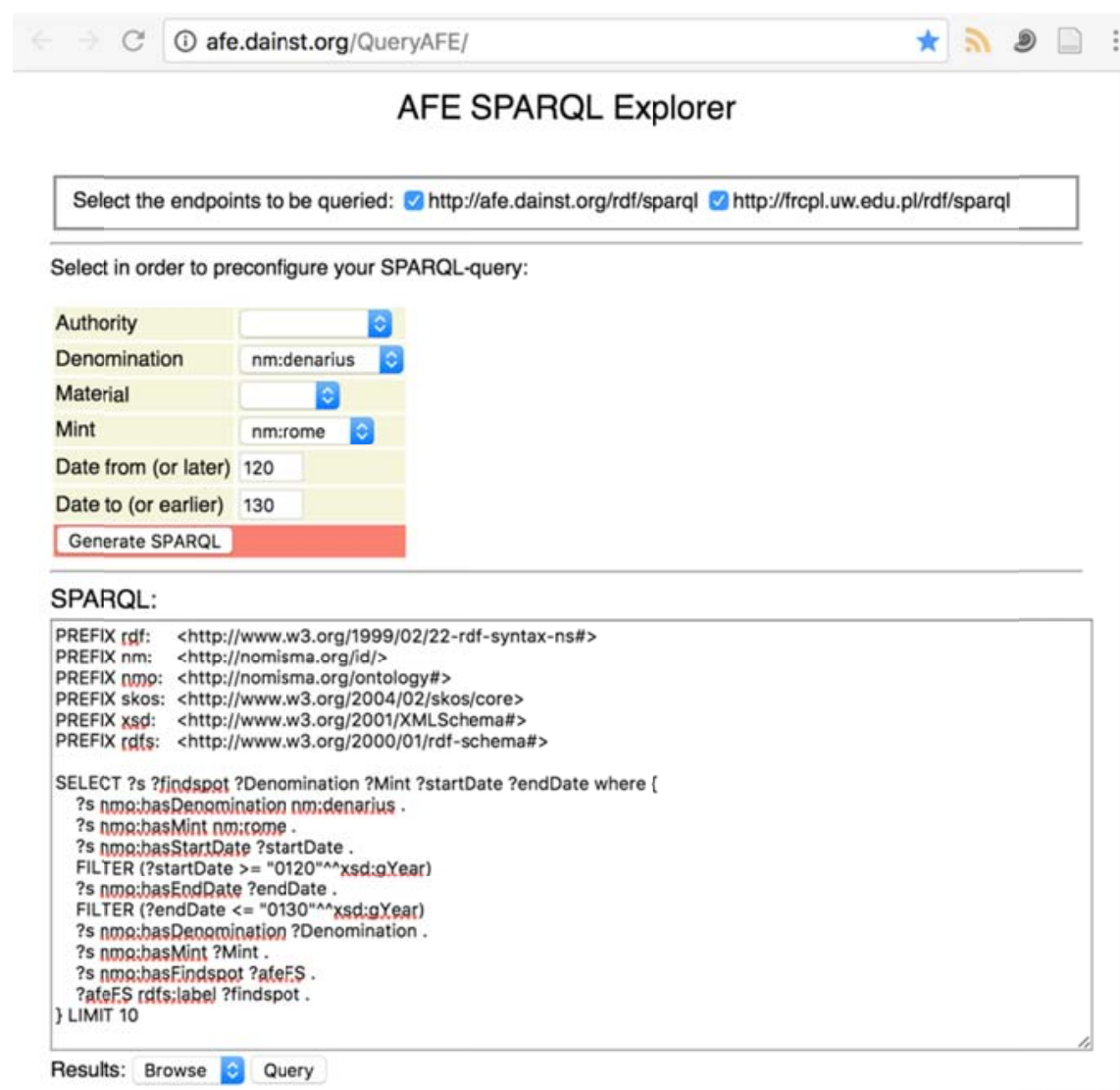

Figure 5. SPARQL-Endpoint pour requêter les bases de données de Francfort et Varsovie (http://afe.fundmuenzen.eu/index.php/sparql-endpoint)

La requête dit : Rechercher des monnaies pour lesquelles

La dénomination est le denier

L'atelier est Rome, et

La monnaie a été frappée entre les années 120 et 130 après J.-C.

Ensuite lister l'atelier, la dénomination et le lieu de trouvaille.

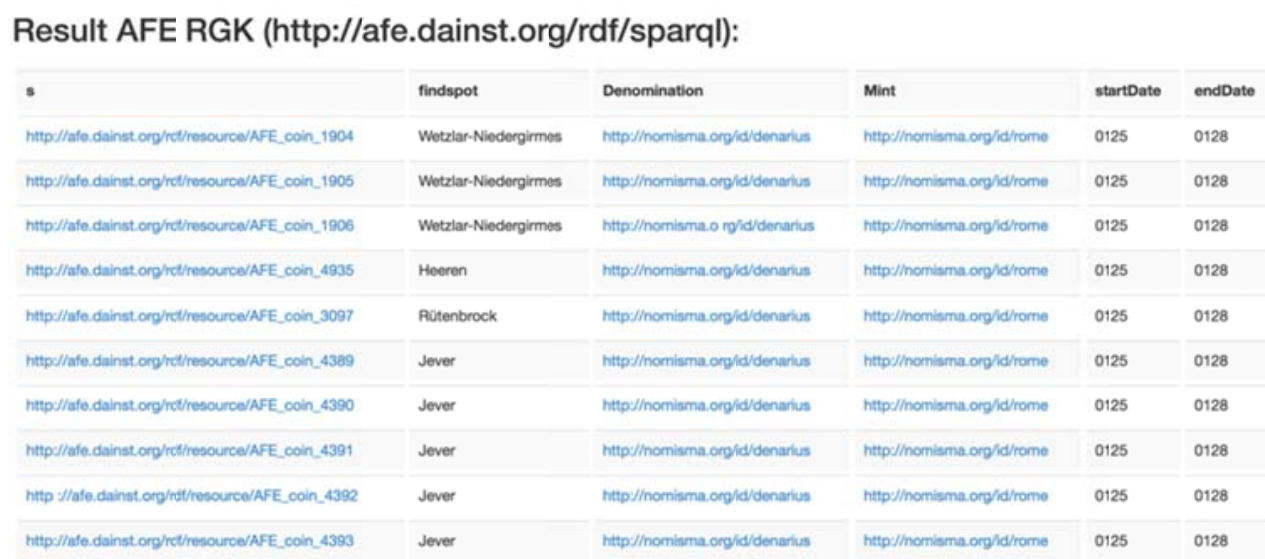

Figure 6. Résultats d'une requête SPARQL. La colonne de gauche donne la liste des liens vers les différentes entrées dans les bases de données AFE. 
Quand la requête est soumise, elle produit une liste de résultats avec les champs demandés. Les entrées individuelles sont liées avec leurs jeux de données dans les bases de données relationnelles (la première colonne dans la figure 6), de la même manière que, dans OCRE, les monnaies sont liées aux sites internet des collections qui les conservent (figure 2).

$\mathrm{Du}$ fait des dimensions limitées de cet article, ceci n'est qu'une très brève démonstration des concepts contenus dans l'utilisation des Linked open data et du web sémantique en numismatique. Des informations plus détaillées, incluant des vidéos, sont proposées sur http://ecfn.fundmuenzen.eu/index.php/downloads. En utilisant les concepts et les outils de nomisma.org, il est donc possible de lier des ressources numismatiques disparates, même lorsqu'elles apparaissent structurées de manière très différente. Le potentiel est énorme et de grandes avancées ont été faites.

\section{Solutions numériques pour monnayages complexes : Online Greek Coinage}

\subsection{Que faire sans grand catalogue de référence}

Le monde grec présente une situation intéressante du fait de son étendue et de sa complexité. Du point de vue de l'étude des monnaies, l'usage est de considérer toute région ayant frappé monnaie entre le VII ${ }^{\mathrm{e}}$ et le $\mathrm{I}^{\text {er }}$ siècle avant $\mathrm{J}$.-C. comme grecque à l'exception du monde celtique, des Parthes et de l'extrême orient. Ceci englobe donc les régions de l'Inde à la Méditerranée occidentale. Sous l'Empire romain, les émissions de la partie orientale du monde grec continuent de porter des inscriptions grecques en Méditerranée orientale, témoignant du maintien de traditions antérieures à la conquête romaine. Ainsi la période chronologique couverte s'étend-elle de la fin du $\mathrm{VII}^{\mathrm{e}}$ s. av J.-C. au $\mathrm{III}^{\mathrm{e}} \mathrm{s}$. ap. J.-C. Le nombre d'ateliers actifs durant cette période est d'environ 1800 d'après les collections du département des Monnaies, médailles et antiques de la $\mathrm{BnF}$, une des collections les plus abondantes au monde.

Il n'existe pas de catalogue général des monnaies grecques en dehors d'un manuel daté de 1911 (Head 1911) qui propose un panorama incomplet mais jamais dépassé. La bibliographie est dispersée à travers les catalogues de collections, ou des corpus dédiés à telle cité ou telle dynastie. Le matériel luimême est d'un accès compliqué : de nombreuses monnaies de bronze conservées dans des musées ou des dépôts archéologiques témoignent d'émissions monétaires inconnues mais ne sont répertoriées dans aucune publication. Cette abondance explique l'absence d'outil central de référence sur le sujet. Il est donc impossible de construire un programme fondé sur un unique catalogue de référence comme l'a fait le programme OCRE. Un catalogue imprimé semblant définitivement impossible - il faudrait publier le premier supplément après la parution du premier volume - la création d'un catalogue de référence en ligne, pouvant être enrichi indéfiniment, paraît être la solution la plus appropriée. C'est ce à quoi se sont attelés plusieurs projets de portails adossés à des projets de recherche ces dernières années.

\subsection{Portails et projets de recherche}

Les portails présentés ici sont dédiés à certains pans de la numismatique grecque mais montrent déjà en germe comment la discipline pourrait se développer en ligne dans les années à venir.

Le projet Kyprios Character est consacré à la monnaie grecque d'argent à Chypre (http://kyprioscharacter.eie.gr/en/). Très classiquement il donne un accès à toutes les collections partenaires proposant des monnaies chypriotes en ligne. Il n'héberge donc aucune image, mais propose pour chaque série, chaque roi, chaque concept, des articles rédigés par des spécialistes du domaine. L'ensemble est construit en utilisant les URIs nomisma pour les concepts grecs.

Un projet du même ordre est consacré aux monnayages thraces par l'académie de Berlin : le Corpus nummorum thracorum (http://www.corpus-nummorum.eu/). L'intérêt du projet est de se fonder aussi 
sur l'importante collection de moulages de monnaies de cette académie. Il montre ainsi comment les ressources mises en ligne par les institutions qui les possèdent peuvent être facilement exploitées de manière croisée.

Ces deux projets se fondent sur les collections déjà disponibles sur le Web. Ces dernières continuent d'être une part minoritaire du matériel disponible dans les musées. A titre d'exemple, seuls la Bibliothèque nationale de France (http://gallica.bnf.fr/html/und/objets/monnaies-grecques) et le musée de l'université d'Harvard (http://www.harvardartmuseums.org/) ont mis en ligne la totalité de leurs collections grecques avec des images. Une partie des collections majeures comme celles du British Museum, de l'American Numismatic Society ou du Münzkabinett de Berlin sont disponibles en ligne. Ces institutions numérisent leurs collections sur fonds propres mais aussi par le biais de financement de programmes de recherche. Grâce à ces programmes, l'exploitation scientifique des collections se fait en même temps que le catalogage, garantissant l'approche la plus pointue de ces objets complexes. C'est le cas par exemple d'OPAL : The Oxford-Paris Alexander Project', co-financé par le Labex les Passés dans le Présent de l'université Paris Nanterre et l'Arts and Humanities Research Council (AHRC) britannique. En un unique programme de 18 mois, les collections de monnaies aux types d'Alexandre le Grand de la Bibliothèque nationale de France et de l'Ashmolean Museum d'Oxford font l'objet d'une campagne de description et de numérisation (Oxford). Chaque monnaie reçoit un identifiant numérique propre et les concepts numismatiques sont affectés d'URIs nomisma. L'objectif du projet est d'ajouter ces collections importantes au portail consacré à la numismatique d'Alexandre Le Grand développé à New York : Pella (http://numismatics.org/pella/). Un colloque est prévu à l'issue de ces travaux, au printemps 2017. Il visera à évaluer les apports de la base de données, ses limites, les outils à développer pour exploiter finement les données en masse ainsi fournies. Il montrera aussi l'importance de ces monnayages pour l'histoire d'Alexandre et sa postérité, ainsi que le rôle de tels outils dans le contexte des pillages archéologiques que connaît aujourd'hui le Proche-Orient.

Le portail Pella est construit sur le modèle des portails romains présentés plus haut puisqu'il existe un catalogue de référence (Price 1991). Mais celui-ci est déjà très largement dépassé : les premiers mois de travail sur le projet OPAL ont montré que 15\% des types monétaires présents dans la collection de Paris n'étaient pas décrits dans Price. A terme, Pella se détachera de ce catalogue et deviendra la référence unique avec cet avantage que de nouveaux types ou variétés découverts pourront y être ajoutés, garantissant ce site contre l'obsolescence inévitable que connaissent les catalogues imprimés.

\subsection{Vers une fédération : Online Greek Coinage}

L'intérêt majeur de ce mouvement de création de catalogues et de portails en ligne est qu'il permet d'envisager la construction d'un corpus des monnaies grecques à échéance raisonnable. Les initiatives prises à Chypre, Berlin, Oxford, Paris ou New York peuvent être fédérées en ligne de même que toutes les initiatives à venir. C'est l'objectif de Online Greek Coinage (OGC : http://www.greekcoinage.org/), soutenu par l'International Numismatic Council (INC, http://www.inc-cin.org/).

Dans la droite ligne du projet nomisma.org, Online Greek Coinage vise à la création d'un vocabulaire standardisé multilingue propre à la numismatique grecque mis à disposition de tous (http://www.greekcoinage.org/thesauri.html) comme le montre ce petit échantillon de la liste de 171 dénominations actuellement disponible.

\footnotetext{
${ }^{1}$ http://actions-recherche.bnf.fr/BnF/anirw3.nsf/IX01/A2015000496_opal-oxford-paris-alexander-project-perspectivestransnationales-a-I-ere-numerique.
} 


\begin{tabular}{|c|c|}
\hline double-litra & An ancient denomination with the value of 2 litrai \\
\hline diobol & An ancient denomination with the value of 2 obols \\
\hline distater & An ancient denomination with the value of 2 staters \\
\hline dodekadrachm & An ancient denomination with the value of 12 drachms \\
\hline
\end{tabular}

Ces listes permettent aussi une désambiguïsation particulièrement utile pour les noms géographiques qui se répètent parfois. La création d'URIs uniques pour les noms de lieux antiques a fait l'objet d'une vaste entreprise américaine aujourd'hui incarnée par le site pleiades.stoa.org. Dans le cadre du projet nomisma.org, des URIs propres ont été attribuées aux ateliers grecs. Ceux-ci ont souvent porté différents noms au cours de l'Antiquité. L'attribution de ces URIs permet aussi de réunir toutes ces identités sans risque de confusion. 


\begin{tabular}{|c|c|c|c|c|c|}
\hline $\begin{array}{c}\text { Antiochei } \\
\text { a ad } \\
\text { Cragum }\end{array}$ & $\begin{array}{c}\text { http://nomisma.org/id/antiocheia_ad_crag } \\
\text { um }\end{array}$ & $\begin{array}{c}\text { http://pleiades.stoa.org/places } / 648 \\
560\end{array}$ & Cilicia & $\begin{array}{c}\text { Antiokhe } \\
\text { ia pros } \\
\text { Kragon }\end{array}$ & \\
\hline $\begin{array}{c}\text { Antiochei } \\
\text { a ad } \\
\text { Cydnum }\end{array}$ & http://nomisma.org/id/tarsus & $\begin{array}{c}\text { http://pleiades.stoa.org/places/648 } \\
789\end{array}$ & Cilicia & $\begin{array}{c}\text { Antiokhe } \\
\text { ia pros } \\
\text { Kydnon }\end{array}$ & Tarsus \\
\hline $\begin{array}{c}\text { Antiochei } \\
\text { a ad } \\
\text { Euphrate } \\
\text { m }\end{array}$ & $\begin{array}{c}\text { http://nomisma.org/id/antiocheia_ad_euph } \\
\text { ratem }\end{array}$ & $\begin{array}{c}\text { http://pleiades.stoa.org/places } / 658 \\
562\end{array}$ & Syria & $\begin{array}{c}\text { Antiokhe } \\
\text { ia pros } \\
\text { Euphrate } \\
\mathrm{n}\end{array}$ & \\
\hline $\begin{array}{c}\text { Antiochei } \\
\text { a ad } \\
\text { Hippum }\end{array}$ & $\begin{array}{c}\text { http://nomisma.org/id/antiocheia_ad_hipp } \\
\text { um }\end{array}$ & $\begin{array}{c}\text { http://pleiades.stoa.org/places } / 678 \\
185\end{array}$ & Syria & $\begin{array}{c}\text { Antiokhe } \\
\text { ia pros } \\
\text { Hippon }\end{array}$ & \\
\hline $\begin{array}{l}\text { Antiochei } \\
\text { a ad } \\
\text { Maeandru } \\
\text { m }\end{array}$ & $\begin{array}{c}\text { http://nomisma.org/id/antiocheia_ad_mae } \\
\text { andrum }\end{array}$ & $\begin{array}{c}\text { http://pleiades.stoa.org/places/638 } \\
748\end{array}$ & Caria & $\begin{array}{c}\text { Antiokhe } \\
\text { ia pros } \\
\text { Maiandr } \\
\text { on }\end{array}$ & \\
\hline $\begin{array}{l}\text { Antiochei } \\
\text { a ad } \\
\text { Pyramum }\end{array}$ & $\begin{array}{c}\text { http://nomisma.org/id/antiocheia_ad_pyra } \\
\text { mum }\end{array}$ & $\begin{array}{c}\text { http://pleiades.stoa.org/places/648 } \\
702\end{array}$ & Cilicia & $\begin{array}{c}\text { Antiokhe } \\
\text { ia pros } \\
\text { Pyramon }\end{array}$ & Magarsa \\
\hline $\begin{array}{c}\text { Antiochei } \\
\text { a ad } \\
\text { Sarum }\end{array}$ & http://nomisma.org/id/adana & $\begin{array}{c}\text { http://pleiades.stoa.org/places } / 648 \\
548\end{array}$ & Cilicia & $\begin{array}{c}\text { Antiokhe } \\
\text { ia pros } \\
\text { Saron }\end{array}$ & Adana \\
\hline $\begin{array}{l}\text { Antiochei } \\
\text { a ad } \\
\text { Taurum }\end{array}$ & & $\begin{array}{c}\text { http://pleiades.stoa.org/places/634 } \\
79235\end{array}$ & Syria & $\begin{array}{c}\text { Antiokhe } \\
\text { ia pros } \\
\text { Tauron }\end{array}$ & \\
\hline $\begin{array}{c}\text { Antiochei } \\
\mathrm{a} \\
\text { (Cebren) }\end{array}$ & http://nomisma.org/id/antiocheia_cebren & $\begin{array}{c}\text { http://pleiades.stoa.org/places } / 550 \\
633\end{array}$ & Troas & $\begin{array}{c}\text { Antiokhe } \\
\text { ia } \\
\text { (Kebren) }\end{array}$ & \\
\hline $\begin{array}{c}\text { Antiochei } \\
\text { a }\end{array}$ & http://nomisma.org/id/antiocheia_pisidia & $\begin{array}{c}\text { http://pleiades.stoa.org/places/609 } \\
307\end{array}$ & Pisidia & $\begin{array}{c}\text { Antiokhe } \\
\text { ia }\end{array}$ & \\
\hline $\begin{array}{l}\text { Antiochei } \\
\text { a ad } \\
\text { Orontem }\end{array}$ & http://nomisma.org/id/antiocheia_syria & $\begin{array}{c}\text { http://pleiades.stoa.org/places } / 658 \\
381\end{array}$ & Syria & $\begin{array}{c}\text { Antiokhe } \\
\text { ia pros } \\
\text { Oronten }\end{array}$ & $\begin{array}{l}\text { Antiokhe } \\
\text { ia pros } \\
\text { Daphnen }\end{array}$ \\
\hline $\begin{array}{l}\text { Antiochia } \\
\text { ad } \\
\text { Callirhoe } \\
\text { m }\end{array}$ & $\begin{array}{c}\text { http://nomisma.org/id/antiochia_ad_callir } \\
\text { hoem }\end{array}$ & $\begin{array}{c}\text { http://pleiades.stoa.org/places/658 } \\
457\end{array}$ & $\begin{array}{l}\text { Mesopota } \\
\text { mia }\end{array}$ & $\begin{array}{c}\text { Antiokhe } \\
\text { ia pros } \\
\text { Kallirhoe } \\
n\end{array}$ & \\
\hline
\end{tabular}


On le voit, Online Greek Coinage n'est pas un manuel ni un guide du monnayage grec, c'est une base de données de référence pensée pour rendre compte de façon systématique de tous les types de monnayages produits dans le monde grec. À terme, bien sûr, l'objectif est de fournir un catalogue exhaustif de toutes ces productions. L'ampleur de la tâche étant immense, l'intention est de débuter ce catalogue, non pas par une extrémité de la Méditerranée, comme cela a été le cas de tous les projets antérieurs, mais par toutes les régions ou toutes les périodes où des programmes de numérisation ou de recherche sont lancés dans le monde. De ce fait, le paysage dessiné par le site est très incomplet (http://www.greekcoinage.org/resources.html). On l'a vu, Chypre, la Thrace, la numismatique d'Alexandre le Grand, sont particulièrement bien étudiées. Mais les projets se multiplient et les grandes collections connaissent un taux de numérisation croissant rapidement. C'est sans aucun doute de leur côté que se situe l'avenir du projet. La numérisation complète des centaines de milliers de monnaies grecques qu'elles conservent, selon des critères de catalogage communs fondés sur le projet nomisma.org, devrait accélérer le processus de fédération en ligne de ces catalogues. À partir d'une telle densité d'informations requêtables à partir d'un seul site, les travaux de recherche seront beaucoup simplifiés : la construction d'un corpus est aujourd'hui encore l'œuvre de plusieurs années de labeur. Il sera alors plus simple de combler les inévitables lacunes laissées par le caractère incomplet des grandes collections et la limitation géographique ou chronologique des programmes de recherche actuels. C'est la clé pour aboutir, enfin, à un catalogue général des monnaies grecques.

\section{Conclusion}

Le Web de données fournit aujourd'hui un outil déterminant pour le traitement de données en grand nombre comme les monnaies. Les nouveaux outils développés selon ces principes transforment déjà en profondeur le travail des spécialistes. Le projet décrit ici pourrait paraitre très prospectif. Il se développe pourtant rapidement.

Les portails de numismatique romaine CHRR et CRRO en tous deux été lancés en 2013. Leur structuration se fonde sur la réflexion portée par Andrew Meadows et Ethan Gruber qui a ensuite conduit au déploiement du projet nomisma.org².

Pour le monde grec, le coup d'envoi a été donné lors d'une rencontre à Berlin en mai 2012 qui a été l'occasion d'un premier contact entre les grandes collections. En avril 2014, deux journées à Paris on réunit douze musées possédant des collections monétaires grecques pour discuter des conditions du développement de leurs catalogues en ligne. En 2016, plusieurs sites sont dédiés à la numismatique grecque et toutes les grandes collections monétaires sont orientées vers le web de données. Le développement de l'ontologie progresse rapidement.

Les mondes médiéval et moderne sont beaucoup moins internationaux : il s'agit de numismatiques nationales, ce qui ralentit la dynamique, la collaboration étant plus limitée. Cependant, les cabinets des médailles de Berlin et Paris travaillent actuellement à une ontologie des monnaies mérovingiennes dont ils conservent les deux principales collections au monde. Par ailleurs, la mise en ligne des collections de ces périodes conduira sans aucun doute à des développements similaires à ceux du monde antique.

\section{Bibliographie}

HEAD, B.V. 1911, Historia Numorum: A Manual of Greek Numismatics ${ }^{2}$, Oxford.

PRICE, M.J. 1991, The Coinage in the Name of Alexander the Great and Philip Arrhidaeus: A British Museum Catalogue, Zürich-London.

\footnotetext{
${ }^{2}$ http://dlib.nyu.edu/awdl/isaw/isaw-papers/7/meadows-gruber/\#p10.
} 